\title{
Prefrontal Hypometabolism in Alzheimer Disease Is Related to Longitudinal Amyloid Accumulation in Remote Brain Regions
}

\author{
Elisabeth Klupp ${ }^{1,2}$, Timo Grimmer ${ }^{2}$, Masoud Tahmasian ${ }^{1,3-5}$, Christian Sorg ${ }^{3,6}$, Igor Yakushev ${ }^{1,3}$, Behrooz H. Yousefi ${ }^{3,7}$, \\ Alexander Drzezga ${ }^{4}$, and Stefan Förster ${ }^{1,3}$ \\ ${ }^{I}$ Department of Nuclear Medicine, Klinikum Rechts der Isar, Technische Universität München, Munich, Germany; ${ }^{2}$ Department \\ of Psychiatry and Psychotherapy, Klinikum Rechts der Isar, Technische Universität München, Munich, Germany; ${ }^{3}$ Technische \\ Universität München Neuroimaging Center (TUM-NIC), Klinikum Rechts der Isar, Technische Universität München, Munich, \\ Germany; ${ }^{4}$ Department of Nuclear Medicine, University Hospital of Cologne, Cologne, Germany; ${ }^{5}$ Department of Neurology, \\ University Hospital of Cologne, Cologne, Germany; ${ }^{6}$ Department of Neuroradiology, Klinikum Rechts der Isar, Technische Universität \\ München, Munich, Germany; and ${ }^{7}$ Pharmaceutical Radiochemistry, Technische Universität München, Garching, Germany
}

In PET studies of patients with Alzheimer disease (AD), prominent hypometabolism can occur in brain regions without major amyloid load. These hypometabolism-only $(\mathrm{HO})$ areas may not be explained easily as a consequence of local amyloid toxicity. The aim of this longitudinal multimodal imaging study was the investigation of locoregional and remote relationships between metabolism in $\mathrm{HO}$ areas and longitudinal amyloid increase in functionally connected brain areas, with a particular focus on intrinsic functional connectivity as a relevant linking mechanism between pathology and dysfunction. Methods: Fifteen $A D$ patients underwent longitudinal examinations with ${ }^{11} \mathrm{C}$-Pittsburgh compound $\mathrm{B}\left({ }^{11} \mathrm{C}-\mathrm{PiB}\right)$ and ${ }^{18} \mathrm{~F}-$ FDG PET (mean follow-up period, 2 y). The peak HO region was identified by the subtraction of equally thresholded statistical T maps (hypometabolism minus amyloid burden), resulting from voxel-based statistical parametric mapping group comparisons between the $A D$ patients and 15 healthy controls. Then functionally connected and nonconnected brain networks were identified by means of seedbased intrinsic functional connectivity analysis of the resting-state functional MRI data of healthy controls. Finally, network-based, regionof-interest-based, and voxel-based correlations were calculated between longitudinal changes of normalized ${ }^{11} \mathrm{C}-\mathrm{PiB}$ binding and ${ }^{18} \mathrm{~F}-\mathrm{FDG}$ metabolism. Results: Positive voxel-based and region-ofinterest-based correlations were demonstrated between longitudinal ${ }^{11} \mathrm{C}$-PiB increases in the HO-connected network, encompassing bilateral temporoparietal and frontal brain regions, and metabolic changes in the peak $\mathrm{HO}$ region as well as locoregionally within several $A D$-typical brain regions. Conclusion: Our results indicate that in $A D$ amyloid accumulation in remote but functionally connected brain regions may significantly contribute to longitudinally evolving hypometabolism in brain regions not strongly affected by local amyloid pathology, supporting the amyloid- and networkdegeneration hypothesis.

Key Words: Alzheimer disease; PET; ${ }^{18} \mathrm{~F}-\mathrm{FDG} ;{ }^{11} \mathrm{C}-\mathrm{PiB}$; rs-fMRI

J Nucl Med 2015; 56:399-404

DOI: 10.2967/jnumed.114.149302

\footnotetext{
Received Oct. 26, 2014; revision accepted Jan. 12, 2015.

For correspondence or reprints contact: Elisabeth Klupp, Ismaninger Strasse 22, 81675 Munich, Germany.

E-mail: Elisabeth.Klupp@gmx.de

Published online Feb. 12, 2015.

COPYRIGHT (c) 2015 by the Society of Nuclear Medicine and Molecular Imaging, Inc.
}

A lzheimer disease (AD) is a progressive neurodegenerative disorder (1). Current hypotheses about the pathogenesis assume that the aggregation of $\beta$-amyloid peptides plays an important role and represents an early event of this disease by leading to synaptic dysfunction and synaptic loss, finally causing neuronal cell death and cognitive decline $(2,3)$. The understanding of causal relations and chronologic sequences of amyloid deposition and neuronal dysfunction in the development of $\mathrm{AD}$ is important for the classification of disease severity and for the evaluation of new treatment approaches. The current study aims at such sequences of patterns of both amyloid plaque deposition and neural dysfunction, with particular focus on intrinsic functional connectivity as a relevant linking mechanism between pathology and dysfunction.

With noninvasive PET biomarkers, such as Pittsburgh compound $\mathrm{B}\left({ }^{11} \mathrm{C}-\mathrm{PiB}\right)$ and ${ }^{18} \mathrm{~F}-\mathrm{FDG}, \mathrm{AD}$-related pathologies, namely fibrillary amyloid burden and hypometabolism, as well as their longitudinal alterations and associations can be investigated in vivo. Compared with healthy subjects, $A D$ patients show specific patterns of reduced ${ }^{18} \mathrm{~F}$-FDG metabolism, as a measure for neuronal dysfunction (4), and increased amyloid deposition (5-7), which are highly overlapping especially in the posterior cingulate cortex (PCC) and parietal and temporal cortical areas (8).

Beyond these commonalities, also discrepancies between the patterns of amyloid-pathology and hypometabolism can be identified in patients with $\mathrm{AD}$ and mild cognitive impairment (MCI), for example, brain regions harboring amyloid burden but normal metabolism and hypometabolic areas without major amyloid deposition (8-10). The absence of hypometabolism in brain areas showing amyloid deposition might be explained by the temporal delay to which hypometabolism follows amyloid deposition. Indeed, a recently published longitudinal study in mildly affected $\mathrm{AD}$ patients demonstrated that the pattern of hypometabolism roughly followed the baseline pattern of amyloid deposition during an observation period of 2 y (10). However, the development of hypometabolism in $\mathrm{AD}$ is most probably more a mixture of phenomena and cannot completely be explained by local amyloid toxicity. Different pathologic processes, such as tau pathology or oxidative stress, may also contribute to the development of neuronal dysfunction $(11,12)$. In the context of a linking mechanism between $\mathrm{AD}$ pathologies and neuronal dysfunction, intrinsic functional connectivity has been 
proposed (13), which is measureable by means of resting-state functional MRI (rs-fMRI). With this method, correlations between spontaneous low-frequency activity fluctuations in the blood-oxygenlevel-dependent (BOLD) signal can be measured to identify synchronously active brain regions. This synchronicity indicates a functional interaction and neuronal communication between these regions (14), which is also related to structural connectivity (15).

By combining rs-fMRI with PET, relations between distribution patterns and quantitative interrelations of $\mathrm{AD}$ pathologies within the structure of intrinsic connectivity networks (ICNs) can be investigated. Indeed, a strong regional overlap between the typical patterns of PET-assessed amyloid deposition and hypometabolism and the patterns of ICNs has already been demonstrated in AD patients. These overlaps were found especially for the default mode network $(13,16)$, one of the most prominent and consistent ICNs associated with the processing of internally focused tasks (17), as well as also for other frontoparietal ICNs (18). Importantly, it also has been demonstrated that $\mathrm{AD}$ and MCI patients show disruptions of functional and structural connectivity within ICNs $(19,20)$ and that even elderly nondemented ${ }^{11} \mathrm{C}$-PiB-positive subjects show associations between hypometabolism, ${ }^{11} \mathrm{C}$-PiB-uptake, and connectivity disruption $(21,22)$.

We hypothesized that hypometabolism in brain regions not strongly affected by local amyloid deposition may be related to longitudinal increases in amyloid deposition in remote but functionally connected brain areas. We assume that an increase of toxic amyloid may contribute to local synaptic dysfunction, leading to reduced neuronal activity and disruption of connectivity. Reduced neuronal activity and disruption of connectivity may lead to a progressively diminished communication within connected brain areas, measurable as hypometabolism there in the absence of prominent local amyloid deposition. The longitudinal measurement of amyloid deposition and hypometabolism was obtained by baseline and follow-up (FU) ${ }^{18} \mathrm{~F}$-FDG and ${ }^{11} \mathrm{C}$-PIB PET imaging during a 2-y time period in a sample of mildly affected AD patients. For measurement of ICNs, rs-fMRI data from elderly healthy controls were obtained at a single time point.

\section{MATERIALS AND METHODS}

\section{Participants}

Similar to a recently published study (23), 15 right-handed patients with an initial diagnosis of mild probable $\mathrm{AD}$, recruited from the outpatient unit for cognitive disorders (Department of Psychiatry and Psychotherapy, Technische Universität München), underwent baseline and FU examinations with ${ }^{18} \mathrm{~F}$-FDG PET, ${ }^{11} \mathrm{C}$-PIB PET, and structural T1-weighted MR imaging with a mean FU period of $27.8 \pm 3.57$ mo. Patients received assessment of apolipoprotein E (APOE) genotype and a standard battery of neuropsychologic tests twice, including the Mini-Mental State Examination (MMSE) (24) and the Clinical Dementia Rating (CDR) scale (25). Fifteen righthanded, cognitively normal (HC1) subjects, recruited by advertisement, served as the control group and underwent the identical testing and imaging procedures once.

For the analysis of physiologic rs-ICNs, we used and analyzed the dataset of a preexisting population of 17 right-handed cognitively normal elderly (HC2) subjects, who underwent an examination with rs-fMRI and structural MR imaging once (Table 1). Before attending, all participants provided written informed consent regarding the scientific evaluation of their data, which was approved by the ethics committee of Technische Universität München and the German radiation protection authority.

\section{Imaging Procedures}

PET Data Acquisition and Analysis. Each AD patient underwent baseline and FU ${ }^{18} \mathrm{~F}-\mathrm{FDG}$ and ${ }^{11} \mathrm{C}-\mathrm{PiB}$ PET imaging, whereas $\mathrm{HC} 1$ subjects were examined once. PET and MR data were acquired and processed using the same tracer-specific protocols and preprocessing steps as published previously $(5,10)$. The methodology of scanner details, PET data evaluation, normalization, statistical group comparisons, and correction for potential influence of regional cortical atrophy are described in the supplemental data (supplemental materials are available at http://jnm.snmjournals.org). For the identification of brain regions showing significant hypometabolism without local amyloid deposition in $\mathrm{AD}$ patients relative to $\mathrm{HC} 1$ subjects, the resulting $\mathrm{T}$ maps from the statistical parametric mapping 5 (SPM5) group comparisons of ${ }^{18} \mathrm{~F}-\mathrm{FDG}$ and ${ }^{11} \mathrm{C}-\mathrm{PiB}$ PET data at FU were overlaid and subtracted using MRIcron (www.sph.sc.edu/comd/rorden/mricron/). We describe the identical method in more detail in a recently published study (23). The identified hypometabolism-only (HO) area was used as a seed region for the calculation of an intrinsic functional connectivity network (HO-ICN) in $\mathrm{HC} 2$ subjects. To ensure that hypometabolism in the HO area occurred still without colocalization of amyloid deposits in AD patients, we used the FU PET T maps for identification.

To quantify longitudinal annual amyloid changes over the observation period, ${ }^{11} \mathrm{C}-\mathrm{PiB}$ annual rate of change (ARC) images were created for every $\mathrm{AD}$ patient in analogy to a procedure previously described by Villain et al. (26), using the formula $\mathrm{FU}{ }^{11} \mathrm{C}-\mathrm{PiB}$ scan minus baseline ${ }^{11} \mathrm{C}-\mathrm{PiB}$ scan divided by $\mathrm{FU}$ duration in years.

These ARC images are supposed to represent differences of amyloid accumulation over time and were used for further analyses. A detailed description is given in the supplemental data.

rs-fMRI Data Acquisition and Analysis. For analysis of intrinsic functional connectivity in $\mathrm{HC} 2$ subjects, MR imaging was performed using the same protocols as published previously (23). Scanner details, acquisition parameters, preprocessing, and analytic steps are described in the supplemental data. To identify the ICN of the HO seed in HC2 subjects, group analyses were performed using the same statistical thresholds as for PET analyses.

\section{Cross-Modality Analyses}

For evaluation of regional and quantitative associations between metabolism and amyloid deposition over time in AD patients, ROIbased and voxelwise correlations between ${ }^{18} \mathrm{~F}-\mathrm{FDG}$ and ${ }^{11} \mathrm{C}-\mathrm{PiB}$ annual changes were calculated. To assess baseline and FU ${ }^{18} \mathrm{~F}-\mathrm{FDG}$ uptake in specific brain regions in $\mathrm{AD}$ patients quantitatively, standardized uptake value ratios (SUVRs) were calculated using the vermis as a reference region. In the same manner as for ${ }^{11} \mathrm{C}-\mathrm{PiB}$, annual changes in ${ }^{18} \mathrm{~F}$-FDG uptake in every patient were calculated using the formula FU ${ }^{18} \mathrm{~F}$-FDG SUVR minus baseline ${ }^{18} \mathrm{~F}$-FDG SUVR divided by FU duration in years.

To ensure comparability between the different PET imaging techniques, the coregistered and spatially normalized ${ }^{18} \mathrm{~F}-\mathrm{FDG}$ images were processed in the same way as the ${ }^{11} \mathrm{C}-\mathrm{PiB}$ ARC images. Longitudinal ${ }^{11} \mathrm{C}-\mathrm{PiB}$ uptake values for specific brain regions were extracted from the ${ }^{11} \mathrm{C}$ $\mathrm{PiB}$ ARC images. Correlations were calculated between annual changes in ${ }^{18} \mathrm{~F}-\mathrm{FDG}$ uptake in the $\mathrm{HO}$ area and changes in ${ }^{11} \mathrm{C}-\mathrm{PiB}$ uptake in the $\mathrm{HO}-\mathrm{ICN}$ as well as locoregionally within $\mathrm{AD}$-typical brain areas.

Whole-Brain Voxelwise Regression and Correlations Between $\mathrm{HO}$ Area and HO-ICN. For the assessment of quantitative interrelations, region-of-interest-based Pearson correlation coefficients were calculated between longitudinal SUVR changes in ${ }^{18} \mathrm{~F}-\mathrm{FDG}$ metabolism in the $\mathrm{HO}$ area and in ${ }^{11} \mathrm{C}-\mathrm{PiB}$ uptake within the HO-ICN in AD patients (significance level of $P<0.05$ [2-tailed]). To avoid autocorrelation, an area with a $20-\mathrm{mm}$ radius around the $\mathrm{HO}$ seed was subtracted from the templates. To examine interrelations between local metabolism in the $\mathrm{HO}$ area and longitudinal amyloid increases over time within the 
TABLE 1

Subjects' Characteristics

\begin{tabular}{|c|c|c|c|c|}
\hline Characteristic & AD patients & $\mathrm{HC} 1$ (PET) & $\mathrm{HC} 2$ (fMRI) & $P(\mathrm{AD}$ vs. $\mathrm{HC} 1 / \mathrm{AD}$ vs. $\mathrm{HC} 2)$ \\
\hline$n$ & 15 & 15 & 17 & \\
\hline Women (\%) & $40 \%$ & $67 \%$ & $59 \%$ & $0.272 / 0.479$, Fisher exact \\
\hline Mean age $(y)$ & $65.00 \pm 8.80$ & $64.60 \pm 5.77$ & $65.05 \pm 5.62$ & $0.884 / 0.982$ \\
\hline \multicolumn{5}{|l|}{ MMSE } \\
\hline Baseline & $23.80 \pm 3.34$ & $29.27 \pm 0.70$ & $29.18 \pm 0.81$ & $0.000 / 0.000$ \\
\hline Follow up & $17.93 \pm 8.44$ & $29.27 \pm 0.70$ & $29.18 \pm 0.81$ & $0.000 / 0.000$ \\
\hline \multicolumn{5}{|l|}{ CDR (global) } \\
\hline \multirow[t]{2}{*}{ Baseline } & 0.50 (Median) & 0.00 (Median) & 0.00 (Median) & 0.000/0.000, Mann-Whitney U \\
\hline & 0.50 (Range) & 0.00 (Range) & 0.50 (Range) & \\
\hline \multirow[t]{2}{*}{ Follow up } & 1.00 (Median) & 0.00 (Median) & 0.00 (Median) & 0.000/0.000, Mann-Whitney U \\
\hline & 1.50 (Range) & 0.00 (Range) & 0.50 (Range) & \\
\hline APOE $\varepsilon 4(+/-)$ & $10 / 5$ & $8 / 7$ & $7 / 10$ & $0.710 / 0.462$, Fisher exact \\
\hline Education (y) & $8.93 \pm 1.58$ & $9.79 \pm 1.72$ & $9.53 \pm 1.55$ & $0.175 / 0.303$ \\
\hline
\end{tabular}

Data are mean \pm SD. $P$ values are results from $t$ tests, unless otherwise noted

APOE $\varepsilon 4(+/-)=$ carrier/noncarrier of APO-E $\varepsilon 4$ allele.

whole brain, voxelwise correlations across AD patients were calculated between mean FU ${ }^{18}$ F-FDG SUVR in the HO seed, regarded as a covariate, and longitudinal ${ }^{11} \mathrm{C}-\mathrm{PiB}$ changes (ARC images) using the multiple-regression-analysis design in SPM5. By this additional analysis, the ROI-based regional correlations between metabolism in the $\mathrm{HO}$ seed and longitudinal ${ }^{11} \mathrm{C}$-PiB changes should be verified using an independent methodology looking for correlations in the whole brain. For these confirmatory analyses, we allowed SPM T maps to be displayed with a liberal threshold of $P$ less than 0.05 uncorrected and a minimum spatial extent of 20 contiguous voxels. The resulting correlation map was overlapped with the $\mathrm{HO} \mathrm{CN}$ to identify intrinsically connected brain regions in which amyloid changes show strong associations with metabolism in the HO seed.

To countercheck our hypothesis, we calculated ROI-based correlations between longitudinal changes in metabolism in the $\mathrm{HO}$ seed and changes in ${ }^{11} \mathrm{C}-\mathrm{PiB}$ uptake within a composite ROI, consisting of bihemispheric neocortical brain areas outside the HO-ICN and within the cerebellar vermis, a brain region typically showing none of the investigated pathologies in sporadic AD. This ROI was created with MRIcron by subtracting the HO-ICN ROI from an anatomic automatic labeling-based whole-brain template (WFU_pickatlas) (27).

Correlations Within AD-Typical Brain Areas. ROI-based correlations between longitudinal SUVR changes in metabolism and in ${ }^{11} \mathrm{C}$ $\mathrm{PiB}$ uptake were calculated locoregionally within brain areas typically affected by AD pathologies. For this purpose, the PCC, precuneus, and whole $\mathrm{HO}-\mathrm{ICN}$, including several AD-typical brain regions, were selected. An overview of the study design is illustrated in Figure 1.

\section{RESULTS}

In $\mathrm{AD}$ patients, mean baseline and FU MMSE scores were significantly lower and CDR scores significantly higher than in $\mathrm{HC1}$ and HC2 subjects $(P<0.001)$. Longitudinal comparison of MMSE scores in $\mathrm{AD}$ patients revealed a significant decrease $(P=0.001)$. There were no significant differences regarding age, sex distribution, and APOE genotype between AD patients and $\mathrm{HC} 1$ or HC2 subjects (Table 1).

\section{${ }^{11} \mathrm{C}-\mathrm{PiB},{ }^{18} \mathrm{~F}-\mathrm{FDG}$ PET, and Functional Connectivity Analyses}

Consistent with the initial clinical diagnosis of mild probable $\mathrm{AD}$, already at baseline all single patients showed typical patterns of hypometabolism and amyloid deposition, whereas $\mathrm{HC} 1$ subjects showed normal cerebral ${ }^{18} \mathrm{~F}-\mathrm{FDG}$-assessed metabolism and no prominent ${ }^{11} \mathrm{C}-\mathrm{PiB}$ binding. The voxel-based group comparisons revealed typical patterns of hypometabolism and ${ }^{11} \mathrm{C}-\mathrm{PiB}$ uptake in $\mathrm{AD}$ patients (baseline and $\mathrm{FU}$ ), with a distinct, regional spread for hypometabolism and a minor spread for amyloid deposition when baseline and FU were compared (Supplemental Fig. 1).

The most conspicuous HO area in our AD group was located in the left middle frontal gyrus (MNI [Montreal Neurologic Institute] coordinates: -30 12 62) (Fig. 2) (23). This HO area was transferred in the the rs-fMRI dataset of $\mathrm{HC} 2$ subjects. Brain regions in which the BOLD signal time courses showed significant positive correlations with the BOLD signal of the HO seed (HO-ICN) were located in the precuneus and frontal, parietal, and temporal cortices of both hemispheres. Less significant correlations were found in occipital, cerebellar, and thalamic areas (Fig. 2).

\section{Cross-Modality Analyses}

The non-hypothesis-driven voxelwise correlation analysis between FU hypometabolism in the HO seed (FU ${ }^{18}$ F-FDG SUVRs) and longitudinal ${ }^{11} \mathrm{C}-\mathrm{PiB}$ increase over time in the whole brain revealed a correlation map, which comprised particularly bihemispheric frontal and parietotemporal brain areas. This correlation map showed a high overlap with the HO-ICN, especially in remote but connected brain areas (e.g., bilateral temporoparietal cortex; Fig. 2). Quantitative ROI-based correlation analyses revealed a significant positive correlation between annual SUVR changes of ${ }^{18} \mathrm{~F}-\mathrm{FDG}$ PETassessed metabolism in the $\mathrm{HO}$ area and ${ }^{11} \mathrm{C}-\mathrm{PiB}$ changes within the remote ICN ( $\mathrm{r}=0.537, P=0.039)$ (Fig. 3). This correlation could be found neither between $\mathrm{HO}$ seed and brain areas outside the HO-ICN ( $\mathrm{r}=0.470, P=0.077$ ) nor between $\mathrm{HO}$ seed and cerebellar vermis ( $\mathrm{r}=0.229, P=0.412$ ) (Supplemental Fig. 2). Significant positive correlations between annual SUVR changes of metabolism and 


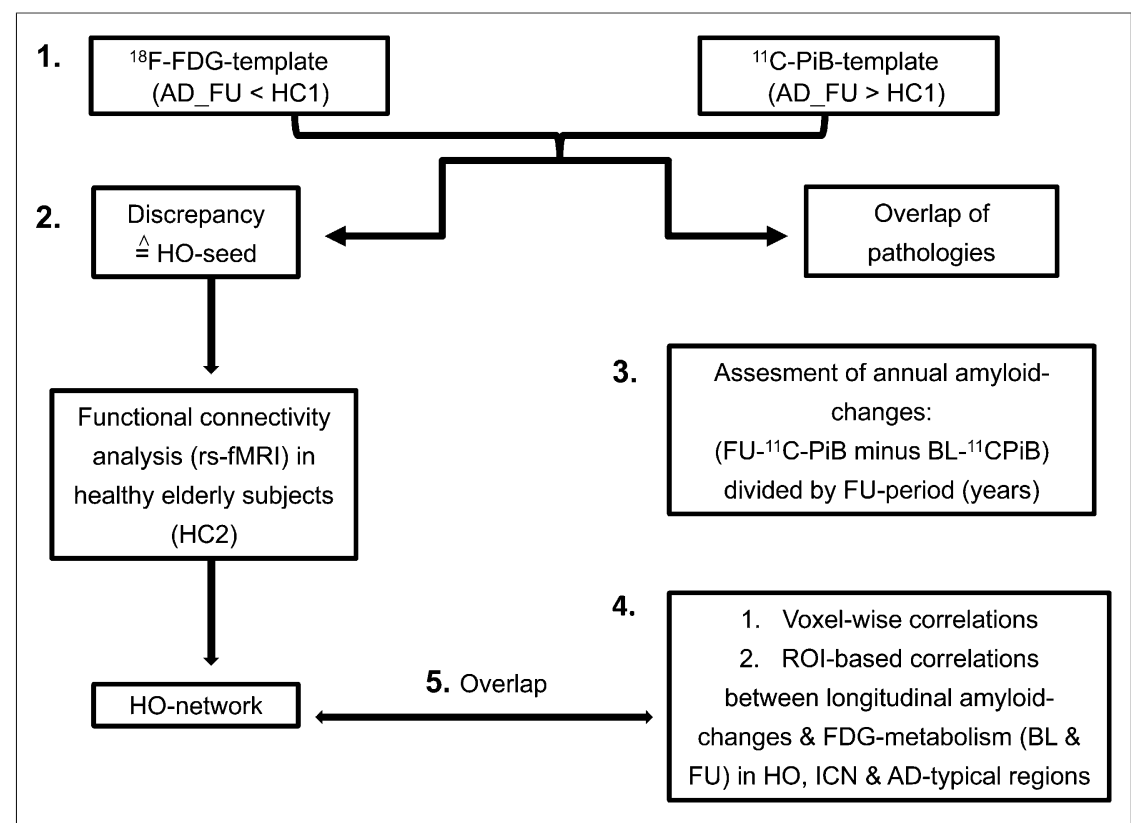

FIGURE 1. 1. First, FU patterns of ${ }^{18} \mathrm{~F}-\mathrm{FDG}$ hypometabolism and ${ }^{11} \mathrm{C}-\mathrm{PiB}$ uptake of $\mathrm{AD}$ patients (compared with $\mathrm{HC} 1$ ) were matched and subtracted to identify regional overlap and discrepancies. 2. Region showing distinct hypometabolism without amyloid pathology ( $\mathrm{HO}$ area) was used as seed region for ROI-based functional connectivity analysis in rs-fMRI dataset of HC2. 3. For assessment of annual changes of amyloid accumulation, ${ }^{11} \mathrm{C}-\mathrm{PiB}$ difference images were created using formula FU PiB minus baseline PiB divided by FU duration in years. 4. ROI-based and voxelwise correlations were calculated between longitudinal amyloid changes and ${ }^{18} \mathrm{~F}-\mathrm{FDG}$ metabolism in $\mathrm{HO}$ area, $\mathrm{HO}-\mathrm{ICN}$, and in AD-typical brain areas. 5. To identify intrinsically connected brain regions in which longitudinal amyloid changes show strong associations with metabolism in $\mathrm{HO}$ seed, resulting correlation map was overlapped with HO-ICN.

${ }^{11} \mathrm{C}$-PiB changes were consistently found also locoregionally within the preselected AD-typical brain regions PCC $(\mathrm{r}=0.677$, $P=0.006)$, precuneus $(\mathrm{r}=0.691, P=0.004)$, and HO-ICN $(\mathrm{r}=$ 0.634, $P=0.011$ ) (Fig. 3; Supplemental Fig. 3).

Furthermore, we were able to significantly link a quantitative measure of hypometabolism (annual SUVR changes) in the HO area to a measure of cognitive decline (baseline and FU MMSE score): baseline: $r=0.662, P=0.007$, and FU: $r=0.695, P=0.004$.

\section{DISCUSSION}

In the present PET study, we investigated sequential associations between longitudinal changes of ${ }^{18} \mathrm{~F}-\mathrm{FDG}$ metabolism and ${ }^{11} \mathrm{C}-\mathrm{PiB}$ deposition: on the one hand between remote but functionally connected brain regions and on the other hand locoregionally within specific brain regions, known to be vulnerable for $\mathrm{AD}$ pathologies. We hypothesized that hypometabolism in brain regions unaffected by local amyloid deposition may be caused by longitudinal increases in amyloid deposition in severely amyloid-affected remote but functionally connected brain areas. For measurement of longitudinal changes in AD pathologies and identification of unaffected, physiologic ICNs, multimodal imaging methods were applied in $\mathrm{AD}$ patients and healthy controls.

We demonstrated a correlation between longitudinal changes in metabolism in a brain region unaffected by fibrillary amyloid and changes in amyloid deposition in functionally connected, strongly amyloid-positive brain regions, indicating a point-to-cause relationship of these 2 pathologies. In contrast, such correlations were not found between longitudinal metabolic changes in this $\mathrm{HO}$ area and changes in amyloid deposition within nonconnected brain regions or a presumably $\mathrm{AD}$ pathology-free brain region, the cerebellar vermis.

Our secondary findings from the PET analyses are consistent with previously published AD studies: AD patients showed, relative to $\mathrm{HC} 1$, typical patterns of amyloid deposition and hypometabolism (5-7) at baseline and FU, confirming the selection of a representative AD patient sample. Furthermore, a distinct regional spread of hypometabolism and a minor spread of amyloid deposition, comparing baseline and $\mathrm{FU}$, were found for $\mathrm{AD}$ patients (28). After superimposing these FU patterns, a pronounced overlap in posterior brain areas and a nonoverlap, particularly in frontal brain regions, was found (8-10). The most conspicuous hypometabolic brain region without local ${ }^{11} \mathrm{C}-\mathrm{PiB}$ uptake (HO area) was identified in the left middle frontal gyrus and used for further analyses (23). The functionally connected $\mathrm{HO}-\mathrm{ICN}$, calculated in $\mathrm{HC} 2$, included the PCC, precuneus, and frontal and parietotemporal areas, which are brain regions with known vulnerability for AD pathologies. The HO-ICN highly overlapped with the calculated correlation map, resulting from our voxelwise whole-brain correlation analysis between metabolism in the $\mathrm{HO}$ seed and longitudinal ${ }^{11} \mathrm{C}-\mathrm{PiB}$ increases, in bihemispheric parietotemporal brain areas. This result supports the view of a point-to-cause relationship between longitudinal increase of toxic amyloid in remote regions and disturbed neuronal activity in a functionally connected region, measured as hypometabolism. The overlapping brain regions and further regions of the HO-ICN are part of the prominent default mode network and represent so-called cortical hub regions, which are highly interconnected brain regions playing an important role in interregional neuronal communication $(13,21,29)$. The hyperactivity of such hub regions during a lifetime could facilitate the later development of $\mathrm{AD}$ pathologies in exactly these brain regions $(16,17)$. Indeed, high activity seems to be related to increased local amyloid production and accumulation, which may have a direct synaptotoxic effect $(30,31)$.

Besides the reported anatomic associations, we also investigated quantitative interrelations between longitudinal SUVR changes in amyloid deposition and in hypometabolism in predefined ROIs in AD patients, namely between $\mathrm{HO}$ area and HO-ICN, between $\mathrm{HO}$ area and nonconnected areas and the cerebellar vermis as well as locoregionally within brain areas typically affected by AD pathologies. A significant positive correlation was found between longitudinal changes in metabolism in the $\mathrm{HO}$ area and longitudinal changes in amyloid deposition within HO-ICN, which could not be found in the investigated control conditions. These results support the view of a point-to-cause relation between increasing amyloid toxicity in a network including AD-typical brain regions and longitudinal metabolic changes in a remote but functionally connected brain region, spared by significant local amyloid deposition and therefore potentially 


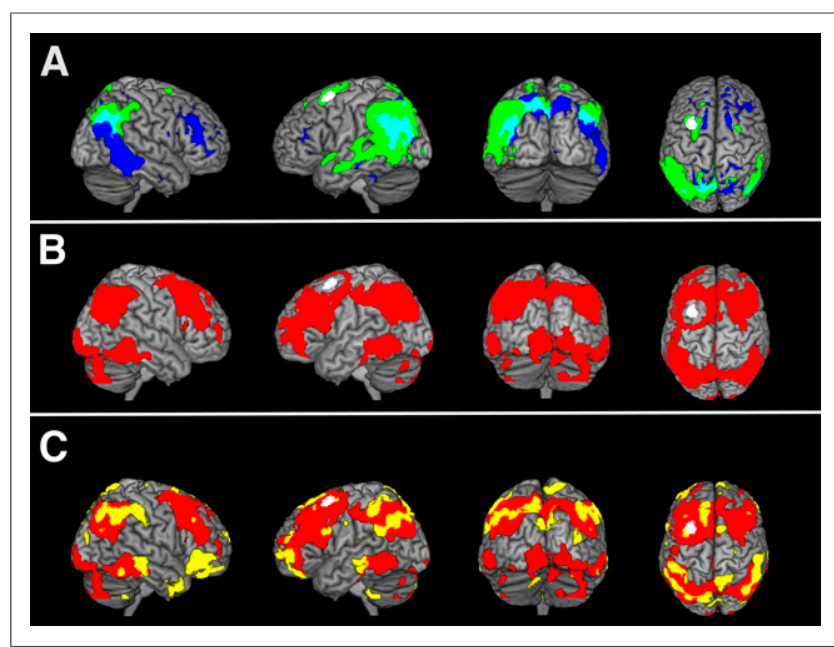

FIGURE 2. (A) Overlap (cyan) and discrepancy (=HO seed; white) between FU amyloid deposition (blue) and FU hypometabolism (green) in $15 \mathrm{AD}$ patients, compared with 15 healthy control subjects (HC1). $P<$ 0.01 , FDR-corrected. (B) ICN (red) based on $\mathrm{HO}$ seed; measured in rsfMRI dataset of 17 control subjects (HC2). Significance threshold $P<$ 0.01 , false-discovery rate (FDR)-corrected. (C) Overlap of voxel-based correlation map (AD patients) between metabolism in $\mathrm{HO}$ and annual accumulation of amyloid in whole brain (yellow) and HO-ICN (HC2; red). Significance thresholds $P<0.05$, uncorrected (correlation-map), and $P<$ 0.01 , FDR-corrected (HO-ICN). Results are displayed on standard MR imaging template.

spared by local amyloid toxicity. Furthermore, we found significant positive correlations between clinical measures of cognitive decline and quantitative changes of metabolism in the $\mathrm{HO}$ area. Locoregionally within the HO-ICN, PCC, and precuneus, even stronger correlations between longitudinal changes in metabolism and in amyloid deposition could be demonstrated in the same direction, fitting into the current literature. In $\mathrm{AD}$ patients, we already found negative correlations between baseline amyloid deposition and longitudinal metabolic changes as well as positive correlations between baseline metabolism and longitudinal amyloid changes in several AD-typical brain areas (32), whereas Cohen et al. demonstrated negative correlations between metabolism and amyloid deposition for AD but positive for MCI patients (33). These findings in MCI patients on a locoregional level and our findings in mildly affected $\mathrm{AD}$ patients on a remote and locoregional level point to an amyloid-mediated compensatory mechanism in preclinical and early clinical stages of $\mathrm{AD}$, for which a potential transient phase of relative upregulation of metabolism (in relation to normalized ${ }^{18} \mathrm{~F}$-FDG SUVRs in AD patients but not to ${ }^{18} \mathrm{~F}$ FDG SUVRs in healthy subjects) might be followed by neuronal breakdown, finally measurable as hypometabolism.

The development, appearance, and interrelations of different AD pathologies within ICNs are in agreement with the recently postulated network degeneration hypothesis (34) indicating that neurodegenerative diseases spread along and affect specific ICNs. This phenomenon has already been suggested for different dementia syndromes (34) and specific clinical AD phenotypes (35). To our best knowledge, the current study is the first one demonstrating longitudinal associations between changes in 2 AD-related pathologies within ICNs. In general, the number of studies dealing with longitudinal relationships between different AD pathologies is limited. A recently published study in hereditary $\mathrm{AD}$ investigated the estimated regional dispersion of amyloid deposition, hypometabolism, and neuronal atrophy in patients with autosomal-dominant AD (presenilin mutation carriers), which averagely show elevated amyloid deposition approximately $15 \mathrm{y}$ before the beginning of disease, whereas hypometabolism and atrophy appeared approximately 10 and 5 y before onset (36). Benzinger et al. (36) demonstrated that most but not all investigated structures with amyloid deposition develop hypometabolism followed by atrophy. Interestingly, in the amyloid-positive precuneus and PCC a hypermetabolic phase before the occurrence of hypometabolism was estimated, fitting into the above-mentioned concept of amyloid-mediated compensatory mechanisms. However, findings from the latter study resulted from estimated longitudinal changes and might not be directly comparable to our observational, truly longitudinal findings in sporadic AD. Tosun et al. demonstrated that increased amyloid deposition in the precuneus and medial temporal regions was associated with atrophy in medial temporal regions in MCI patients (37). In a similar longitudinal study, Villain et al. showed that atrophy in the hippocampal area in MCI patients may lead to disruption of the structural integrity of white matter cingulum bundle fibers, which itself may cause hypometabolism in the PCC (38). Consequently, besides the deposition of toxic amyloid, there seem to exist additional pathomechanisms leading to neuronal dysfunction; however, the interrelations are complex and need to be further evaluated in a longitudinal fashion in larger cohorts of preclinical $\mathrm{AD}$ patients with standardized multimodal PET/MR imaging protocols applied.

There are several limitations to our study. Because of the small sample size, differences from the general population of $\mathrm{AD}$ patients may be expected and our findings may not be generalizable. It is important to mention that our results should be considered as exemplary and need to be reproduced in larger samples in future studies.

Because of different methodologic sensitivities, it may be problematic to compare the results acquired from different imaging techniques. The identified HO area may not be completely free of any amyloid deposition and should be considered as an exemplary region with peak $\mathrm{HO}$ in the investigated $A D$ group because distinct parts of the frontal cortex are typically reported as amyloid-positive brain regions $(5,10)$. Moreover, 
with ${ }^{11} \mathrm{C}-\mathrm{PiB}$ only fibrillary amyloid deposits can be targeted and the probably more toxic soluble amyloid oligomeres cannot be visualized by any of the currently available amyloid PET imaging agents.

\section{CONCLUSION}

Positive quantitative correlations were demonstrated between longitudinal changes of amyloid and hypometabolism in $\mathrm{AD}$ patients. These correlations were found between remote brain regions (HO-ICN and $\mathrm{HO}$ seed) and locoregionally within $\mathrm{AD}$-typical brain regions. The results of this longitudinal study indicate that in $\mathrm{AD}$ amyloid accumulation in remote but functionally connected brain regions may significantly contribute to longitudinally evolving hypometabolism in brain regions not strongly affected by local amyloid pathology, supporting the amyloid- and network-degeneration hypothesis.

\section{DISCLOSURE}

The costs of publication of this article were defrayed in part by the payment of page charges. Therefore, and solely to indicate this fact, this article is hereby marked "advertisement" in accordance with 18 USC section 1734 . This work was supported in part by the German Research Foundation (DFG) (DR 445/4-1, DR 445/5-2, and FO 886/1-1). Alexander Drzezga is acting as consultant for GE Healthcare, Avid/Lilly \& Piramal. Stefan Förster is acting as consultant for GE Healthcare and Piramal. No other potential conflict of interest relevant to this article was reported.

\section{ACKNOWLEDGMENTS}

We are thankful to the participants of the study and the staff of the Departments of Nuclear Medicine, Psychiatry and Neuroradiology for their help in recruitment and data collection.

\section{REFERENCES}

1. Braak E, Griffing K, Arai K, Bohl J, Bratzke H, Braak H. Neuropathology of Alzheimer's disease: what is new since A. Alzheimer? Eur Arch Psychiatry Clin Neurosci. 1999;249(suppl 3):14-22.

2. Hardy J, Selkoe DJ. The amyloid hypothesis of Alzheimer's disease: progress and problems on the road to therapeutics. Science. 2002;297:353-356.

3. Walsh DM, Selkoe DJ. Deciphering the molecular basis of memory failure in Alzheimer's disease. Neuron. 2004;44:181-193.

4. Magistretti PJ, Pellerin L. Cellular mechanisms of brain energy metabolism and their relevance to functional brain imaging. Philos Trans $R$ Soc Lond B Biol Sci. 1999;354:1155-1163.

5. Drzezga A, Grimmer T, Henriksen G, et al. Imaging of amyloid plaques and cerebral glucose metabolism in semantic dementia and Alzheimer's disease. Neuroimage. 2008;39:619-633.

6. Klunk WE, Engler H, Nordberg A, et al. Imaging brain amyloid in Alzheimer's disease with Pittsburgh Compound-B. Ann Neurol. 2004;55:306-319.

7. Minoshima S, Giordani B, Berent S, Frey KA, Foster NL, Kuhl DE. Metabolic reduction in the posterior cingulate cortex in very early Alzheimer's disease. Ann Neurol. 1997;42:85-94.

8. Edison P, Archer HA, Hinz R, et al. Amyloid, hypometabolism, and cognition in Alzheimer disease: an $\left[{ }^{11} \mathrm{C}\right] \mathrm{PIB}$ and $\left[{ }^{18} \mathrm{~F}\right] \mathrm{FDG}$ PET study. Neurology. 2007;68: 501-508.

9. Li Y, Rinne JO, Mosconi L, et al. Regional analysis of FDG and PIB-PET images in normal aging, mild cognitive impairment, and Alzheimer's disease. Eur J Nucl Med Mol Imaging. 2008;35:2169-2181.

10. Förster S, Grimmer T, Miederer I, et al. Regional expansion of hypometabolism in Alzheimer's disease follows amyloid deposition with temporal delay. Biol Psychiatry. 2012;71:792-797.

11. Goedert M, Spillantini MG, Crowther RA. Tau proteins and neurofibrillary degeneration. Brain Pathol. 1991;1:279-286.

12. Su B, Wang X, Nunomura A, et al. Oxidative stress signaling in Alzheimer's disease. Curr Alzheimer Res. 2008;5:525-532.
13. Buckner RL, Sepulcre J, Talukdar T, et al. Cortical hubs revealed by intrinsic functional connectivity: mapping, assessment of stability, and relation to Alzheimer's disease. J Neurosci. 2009;29:1860-1873.

14. Fox MD, Raichle ME. Spontaneous fluctuations in brain activity observed with functional magnetic resonance imaging. Nat Rev Neurosci. 2007;8:700-711.

15. van den Heuvel M, Mandl R, Luigjes J, Hulshoff Pol H. Microstructural organization of the cingulum tract and the level of default mode functional connectivity. J Neurosci. 2008;28:10844-10851.

16. Buckner RL, Snyder AZ, Shannon BJ, et al. Molecular, structural, and functional characterization of Alzheimer's disease: evidence for a relationship between default activity, amyloid, and memory. J Neurosci. 2005;25:7709-7717.

17. Buckner RL, Andrews-Hanna JR, Schacter DL. The brain's default network: anatomy, function, and relevance to disease. Ann N Y Acad Sci. 2008;1124:1-38.

18. Lehmann M, Ghosh PM, Madison C, et al. Diverging patterns of amyloid deposition and hypometabolism in clinical variants of probable Alzheimer's disease. Brain. 2013;136:844-858

19. Hahn K, Myers N, Prigarin S, et al. Selectively and progressively disrupted structural connectivity of functional brain networks in Alzheimer's disease: revealed by a novel framework to analyze edge distributions of networks detecting disruptions with strong statistical evidence. Neuroimage. 2013;81:96-109.

20. Greicius MD, Srivastava G, Reiss AL, Menon V. Default-mode network activity distinguishes Alzheimer's disease from healthy aging: evidence from functional MRI. Proc Natl Acad Sci USA. 2004;101:4637-4642.

21. Drzezga A, Becker JA, Van Dijk KR, et al. Neuronal dysfunction and disconnection of cortical hubs in non-demented subjects with elevated amyloid burden. Brain. 2011;134:1635-1646.

22. Sperling RA, Laviolette PS, O'Keefe K, et al. Amyloid deposition is associated with impaired default network function in older persons without dementia. Neuron. 2009;63:178-188.

23. Klupp E, Forster S, Grimmer T, et al. In Alzheimer's disease, hypometabolism in low-amyloid brain regions may be a functional consequence of pathologies in connected brain regions. Brain Connect. 2014;4:371-383.

24. Folstein MF, Folstein SE, McHugh PR. "Mini-mental state": a practical method for grading the cognitive state of patients for the clinician. J Psychiatr Res. 1975;12:189-198.

25. Morris JC. The clinical dementia rating (CDR): current version and scoring rules. Neurology. 1993;43:2412-2414.

26. Villain N, Chetelat G, Grassiot B, et al. Regional dynamics of amyloid-beta deposition in healthy elderly, mild cognitive impairment and Alzheimer's disease: a voxelwise PiB-PET longitudinal study. Brain. 2012;135:2126-2139.

27. Maldjian JA, Laurienti PJ, Kraft RA, Burdette JH. An automated method for neuroanatomic and cytoarchitectonic atlas-based interrogation of fMRI data sets. Neuroimage. 2003;19:1233-1239.

28. Kadir A, Almkvist O, Forsberg A, et al. Dynamic changes in PET amyloid and FDG imaging at different stages of Alzheimer's disease. Neurobiol Aging. 2012;33:198 e191-114

29. Tomasi D, Volkow ND. Functional connectivity density mapping. Proc Natl Acad Sci USA. 2010;107:9885-9890.

30. Cirrito JR, Yamada KA, Finn MB, et al. Synaptic activity regulates interstitial fluid amyloid-beta levels in vivo. Neuron. 2005;48:913-922.

31. Kamenetz F, Tomita T, Hsieh H, et al. APP processing and synaptic function. Neuron. 2003;37:925-937.

32. Förster S, Yousefi BH, Wester HJ, et al. Quantitative longitudinal interrelationships between brain metabolism and amyloid deposition during a 2-year followup in patients with early Alzheimer's disease. Eur J Nucl Med Mol Imaging. 2012;39:1927-1936.

33. Cohen AD, Price JC, Weissfeld LA, et al. Basal cerebral metabolism may modulate the cognitive effects of Abeta in mild cognitive impairment: an example of brain reserve. J Neurosci. 2009;29:14770-14778.

34. Seeley WW, Crawford RK, Zhou J, Miller BL, Greicius MD. Neurodegenerative diseases target large-scale human brain networks. Neuron. 2009;62:42-52.

35. Lehmann M, Madison CM, Ghosh PM, et al. Intrinsic connectivity networks in healthy subjects explain clinical variability in Alzheimer's disease. Proc Natl Acad Sci USA. 2013;110:11606-11611.

36. Benzinger TL, Blazey T, Jack CR Jr, et al. Regional variability of imaging biomarkers in autosomal dominant Alzheimer's disease. Proc Natl Acad Sci USA. 2013;110:E4502-E4509.

37. Tosun D, Schuff N, Mathis CA, Jagust W, Weiner MW. Alzheimer's disease neuroimaging I: spatial patterns of brain amyloid-beta burden and atrophy rate associations in mild cognitive impairment. Brain. 2011;134:1077-1088.

38. Villain N, Fouquet M, Baron JC, et al. Sequential relationships between grey matter and white matter atrophy and brain metabolic abnormalities in early Alzheimer's disease. Brain. 2010;133:3301-3314. 